\title{
Thermoelectric Properties of P-Type Heusler Compounds $\left(\mathrm{Fe}_{2-x} \mathrm{Co}_{x}\right)\left(\mathrm{V}_{1-y} \mathrm{Ti}_{y}\right) \mathrm{Al}$
}

\author{
Hiroyuki Nakayama*1, Naoki Ide and Yoichi Nishino*2 \\ Department of Materials Science and Engineering, Nagoya Institute of Technology, Nagoya 466-8555, Japan
}

We report on the effect of $\mathrm{Co}$ and $\mathrm{Ti}$ co-doping on the thermoelectric behavior of $\mathrm{Fe}_{2} \mathrm{VAl}$ based alloys. The doped alloys, $\left(\mathrm{Fe}_{2-x} \mathrm{Co}_{x}\right)\left(\mathrm{V}_{1-y} \mathrm{Ti}_{y}\right) \mathrm{Al}$, with compositions $x=0.01-0.07$ and $y=0.04-0.20$ exhibit a p-type thermoelectric property when the total valence electron number is below 24. The co-doping of cobalt and titanium is believed to cause a substantial shift of the Fermi level from the center of the pseuodgap to a lower energy position. In particular, the alloy with $x=0.03$ and $y=0.10$ exhibits a p-type thermoelectric behavior with a large Seebeck coefficient of $S=85 \mu \mathrm{V} / \mathrm{K}$ as well as a low electrical resistivity of $\rho=2.3 \mu \Omega \mathrm{m}$ at $400 \mathrm{~K}$, so that the power factor, $P=S^{2} / \rho$, reaches $3.2 \times 10^{-3} \mathrm{~W} / \mathrm{m} \mathrm{K}^{2}$. The power factor is larger than that of the Ti doped $\mathrm{Fe}_{2} \mathrm{VAl}$ alloy so far reported. The high power factor caused by the co-doping of $\mathrm{Co}$ and Ti might be due to a modification in the band structure on the valence band side around the Fermi level. The co-doping of $\mathrm{Co}$ and $\mathrm{Ti}$ is also effective in reducing the thermal conductivity, mainly due to phonon scattering. [doi:10.2320/matertrans.MRA2008078]

(Received March 3, 2008; Accepted May 21, 2008; Published July 16, 2008)

Keywords: Heusler alloy, Seebeck coefficient, pseudogap, density of states (DOS), thermoelectric material

\section{Introduction}

The Heusler-type intermetallic compound $\mathrm{Fe}_{2} \mathrm{VAl}$ has attractive attention because of the presence of semiconductor-like temperature dependence of electrical resistivity, and the existence of a steep pseudogap of $\sim 0.2 \mathrm{eV}$ in width at the Fermi level in the density of states (DOS) structure. ${ }^{1)}$ The pseudogap $\mathrm{Fe}_{2} \mathrm{VAl}$ system is a new candidate for thermoelectric material, because of the occurrence of a large Seebeck coefficient by fourth element doping or off-stoichiometry. $^{2-10)}$ The Seebeck coefficient, $S$, at a temperature, $T$, in metallic materials is expressed by ${ }^{11}$ )

$$
S(T)=-\frac{\pi^{2}}{3} \frac{k_{\mathrm{B}}^{2} T}{e} \frac{1}{N\left(E_{\mathrm{F}}\right)}\left[\frac{\partial N(E)}{\partial E}\right]_{E=E_{\mathrm{F}}}
$$

where $N\left(\mathrm{E}_{\mathrm{F}}\right)$ is the DOS at the Fermi level, $E_{\mathrm{F}}$. According to eq. (1), the Seebeck coefficient is proportional to the inverse of the DOS and the differential of the DOS with respect to $E, \partial N(E) / \partial E$, and the sign is opposite to that for $\partial N(E) / \partial E$. Thus, the shift of the Fermi level by doping or off-stoichiometry causes an enhancement in the Seebeck coefficient, because the DOS of the $\mathrm{Fe}_{2} \mathrm{VAl}$ alloy sharply changes in both sides of the pseudogap. In addition, Nishino et al. have reported that change in the Seebeck coefficient of fourth element doped alloys at a constant temperature exhibits a rigid-band-like behavior irrespective of the doping elements. $^{12)}$ The band calculation clarified that the iron partial DOS is majority in the valence band side, and both iron and vanadium partial DOSs are dominant in the conduction band in the vicinity of the Fermi level. ${ }^{1)}$ In previous studies, ${ }^{4-6,8,9)}$ the doping was carried out into the vanadium site in the case of the p-type material, but into one of the iron, vanadium and aluminum sites in the n-type material, so that the doping effect on the DOS structure at the Fermi level might be small. Therefore, the rigid-band-like behavior was observed in the fourth element doped alloys.

\footnotetext{
${ }^{* 1}$ Present address: National Institute of Advanced Industrial Science and Technology

${ }^{* 2}$ Corresponding author, E-mail: nishino@nitech.ac.jp
}

In other word, the maximum Seebeck coefficient of $\mathrm{Fe}_{2} \mathrm{VAl}$ based thermoelectric materials is governed by the shape of the DOS structure in the $\mathrm{Fe}_{2} \mathrm{VAl}$ alloy independent of the fourth doping elements. The rigid-band-like behavior is one of issues in the $\mathrm{Fe}_{2} \mathrm{VAl}$ based thermoelectric materials for further improvement of the Seebeck coefficient.

Other issues in the $\mathrm{Fe}_{2} \mathrm{VAl}$ based thermoelectric materials are a smaller absolute value of the Seebeck coefficient for the p-type materials than that for the n-type materials, in addition to restricted doping elements to obtain the p-type property. The p-type thermoelectric behavior has been reported only in the $\mathrm{Ti}$ or $\mathrm{Re}$ doped alloys, and their Seebeck coefficient is about $90 \mu \mathrm{V} / \mathrm{K}$ or less. ${ }^{4,10)}$ In contrast, the absolute value of the Seebeck coefficient of the n-type materials reaches $120 \mu \mathrm{V} / \mathrm{K}$ and above by the fourth element doping. ${ }^{4-6,8)}$ The rigid-band-like behavior stands in the fourth element doped $\mathrm{Fe}_{2} \mathrm{VAl}$ based alloys as mentioned above, so that the change in the DOS structure will be necessary for further improvement of the Seebeck coefficient in the p-type materials, namely both a small DOS and a large value of $\partial N(E) / \partial E$ at the Fermi level are required. We expect that the fifth element doping into the iron site causes a significant change in the DOS structure and that the co-doping of $\mathrm{Co}$ and $\mathrm{Ti}$ is effective in obtaining good thermoelectric behavior. In this study, p-type $\left(\mathrm{Fe}_{2-x} \mathrm{Co}_{x}\right)$ $\left(\mathrm{V}_{1-y} \mathrm{Ti}_{y}\right) \mathrm{Al}$ alloys are fabricated and their thermoelectric properties are assessed.

\section{Experimental Procedure}

Alloy ingots of $\left(\mathrm{Fe}_{2-x} \mathrm{Co}_{x}\right)\left(\mathrm{V}_{1-y} \mathrm{Ti}_{y}\right) \mathrm{Al}$ with $x=0.01$, 0.03 and 0.07 and $y=0.06-0.20$ were prepared by arc melting. The total valence electron number was adjusted to be less than 24 so as to obtain the p-type properties. The source materials $(99.99 \% \mathrm{Fe}, 99.99 \% \mathrm{Al}, 99.9 \% \mathrm{~V}, 99.9 \%$ $\mathrm{Co}$ and $99.9 \% \mathrm{Ti}$ ) were weighted accurately, and then they were melted together. The once melted ingots were flipped and then melted again: this process was repeated three times at least for obtaining homogeneous ingots. The alloy ingots were homogenized at $1273 \mathrm{~K}$ for $172.8 \mathrm{ks}$ in vacuum, 
and then furnace cooled. The homogenized ingots were mechanically formed for powder X-ray diffraction (XRD), Seebeck coefficient $\left(0.5 \times 0.5 \times 5 \mathrm{~mm}^{3}\right)$, electrical resistivity $\left(1 \times 1.5 \times 15 \mathrm{~mm}^{3}\right)$ and thermal conductivity $(3.5 \times$ $3.5 \times 4 \mathrm{~mm}^{3}$ ) measurements. The specimens were annealed at $1273 \mathrm{~K}$ for $3.6 \mathrm{ks}$ and subsequently aged at $673 \mathrm{~K}$ for $14.4 \mathrm{ks}$ in a sealed quartz tube followed by furnace cooling. The total weight loss after melting and heat treatments was less than $0.1 \%$, so that the nominal alloy composition is used in this study.

XRD patterns were obtained using RINT 2100 (Rigaku Co.) with $\mathrm{Cu} \mathrm{K} \alpha$ radiation. The Seebeck coefficient was measured by SB-100 (MMR Technologies Inc.) in the temperature range of $100-400 \mathrm{~K}$. The electrical resistivity was measured by 4-probe method under $100 \mathrm{~mA}$ direct current in the temperature range of $77-1250 \mathrm{~K}$. The thermal conductivity measurement was carried out by a longitudinal steady-state method using a reference material of $\mathrm{Al}_{2} \mathrm{O}_{3}$ $(\kappa=20 \mathrm{~W} / \mathrm{m} \mathrm{K})$ at $300 \mathrm{~K}$ in vacuum.

\section{Results}

Figure 1 shows the XRD results of the $\left(\mathrm{Fe}_{2-x} \mathrm{Co}_{x}\right)$ $\left(\mathrm{V}_{1-y} \mathrm{Ti}_{y}\right) \mathrm{Al}$ alloys with $x=0.03$ and $y=0.06-0.20$. The peaks corresponding to the Heusler structure are seen, but other peaks due to a secondary phase are not detected. Other samples with different Co compositions ( $x=0.01$ and 0.07$)$ showed almost the same results as in Fig. 1, that is only peaks corresponding to the Heusler structure were detected. Unfortunately, the degree of order of the alloys was not determined, because the scattering factors of iron and vanadium are almost the same as those of cobalt and titanium, respectively. However, the intensity ratios of (111) to (220) in each composition are very close to the theoretical

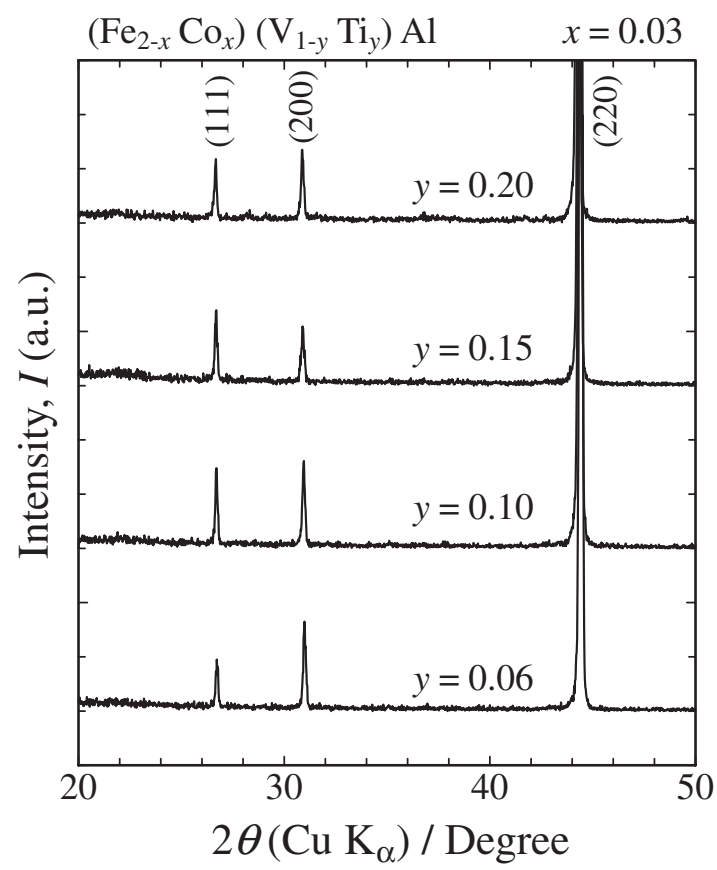

Fig. 1 XRD patterns in $\left(\mathrm{Fe}_{2-x} \mathrm{Co}_{x}\right)\left(\mathrm{V}_{1-y} \mathrm{Ti}_{y}\right) \mathrm{Al}$ alloys with $x=0.03$ and $y=0.06-0.20$.

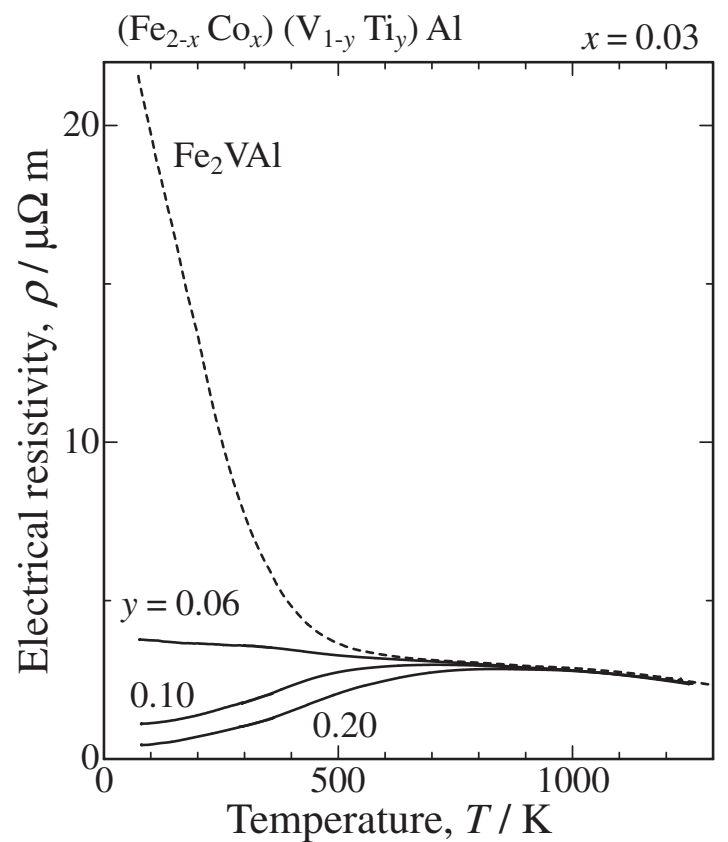

Fig. 2 Temperature dependence of electrical resistivity in $\left(\mathrm{Fe}_{2-x} \mathrm{Co}_{x}\right)$ $\left(\mathrm{V}_{1-y} \mathrm{Ti}_{y}\right) \mathrm{Al}$ alloys with $x=0.03$ and $y=0.06-0.20$. The electrical resistivity of $\mathrm{Fe}_{2} \mathrm{VAl}$ alloy is shown by the broken line.

value: the theoretical intensity ratio is about 0.04 , and the actual intensity ratios are in the range of $0.05-0.06$. Therefore, the degree of order of the alloys used in this study is expected to be very high.

The changes in the electrical resistivity of the $\left(\mathrm{Fe}_{2-x} \mathrm{Co}_{x}\right)$ $\left(\mathrm{V}_{1-y} \mathrm{Ti}_{y}\right) \mathrm{Al}$ alloys with $x=0.03$ and $y=0.06-0.20$ as a function of temperature are shown in Fig. 2. The stoichiometric $\mathrm{Fe}_{2} \mathrm{VAl}$ alloy, as shown by the broken line, exhibits a semiconductor-like dependence on temperature. At low temperatures, the resistivity is extremely high, i.e. $21.3 \mu \Omega \mathrm{m}$ at $77 \mathrm{~K}$, as compared with that for conventional metals, and it decreases remarkably with increasing temperature. The $\mathrm{Co}$ and $\mathrm{Ti}$ doping leads to a significant decrease in the electrical resistivity in the temperature range of $77-900 \mathrm{~K}$. The resistivity at $77 \mathrm{~K}$ is $0.44 \mu \Omega \mathrm{m}$ in the alloy with $x=0.03$ and $y=0.20$, which is only $2 \%$ of that for the stoichiometric $\mathrm{Fe}_{2} \mathrm{VAl}$ alloy. The excess Ti doping $(y>0.10)$ causes a change in the dependence on temperature from semiconductor-like to metallic in the temperature range of 77-900 K. Although the resistivity changes in the low temperature range depend upon the alloy composition, those above $900 \mathrm{~K}$ almost coincide with each other, irrespective of the alloy compositions. In the case of other Co compositions, the trend of the temperature dependence of the electrical resistivity is almost the same as that in Fig. 2 .

Figure 3 shows the temperature dependence of the Seebeck coefficient in (a) $x=0.01$, (b) 0.03 and (c) 0.07 alloys, and for comparison, the Seebeck coefficient of the $\mathrm{Fe}_{2} \mathrm{VAl}$ alloy is plotted by the double circles in Fig. 3(a). The valence electron numbers, $n$, are also indicated below the respective compositions for comparison between the alloys having the same valence electron numbers but the different Co compositions. It is clear that the Seebeck coefficient is improved by the Co and Ti co-doping as compared with that 

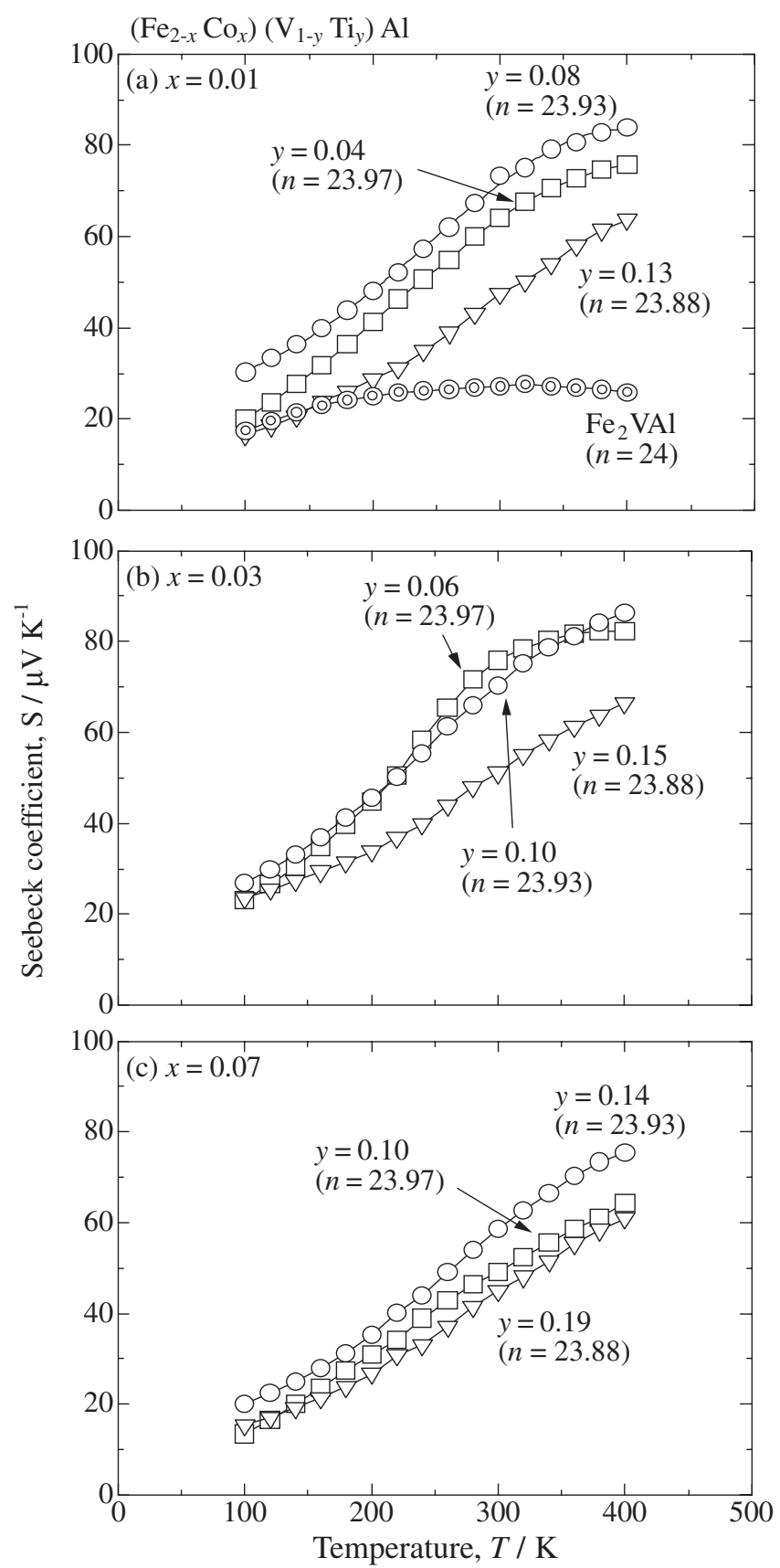

Fig. 3 Temperature dependence of Seebeck coefficient in $\left(\mathrm{Fe}_{2-x} \mathrm{Co}_{x}\right)$ $\left(\mathrm{V}_{1-y} \mathrm{Ti}_{y}\right) \mathrm{Al}$ alloys with composition of (a) $x=0.01$, (b) 0.03 and (c) 0.07 : the valence electron numbers, $n$, are also shown under the compositions. The Seebeck coefficient of $\mathrm{Fe}_{2} \mathrm{VAl}$ alloy is plotted by the double circles in Fig. 3(a).

of the $\mathrm{Fe}_{2} \mathrm{VAl}$ alloy. It is of particular interest to compare the Seebeck coefficient among the alloys with the same valence electron number $(n=23.93)$ at $400 \mathrm{~K}: 83 \mu \mathrm{V} / \mathrm{K}$ for $x=0.01$, $85 \mu \mathrm{V} / \mathrm{K}$ for $x=0.03$ and $75 \mu \mathrm{V} / \mathrm{K}$ for $x=0.07$. There is a relatively large difference in the Seebeck coefficient between the alloys with $x=0.03$ and 0.07 , so that the DOS structure is expected to be different significantly between those alloys. Moreover, the observed Seebeck coefficient of $85 \mu \mathrm{V} / \mathrm{K}$ in the alloy with $x=0.03$ and $y=0.1$ is slightly smaller than $90 \mu \mathrm{V} / \mathrm{K}$ in the $\left(\mathrm{Fe}_{2-x} \mathrm{Re}_{x}\right) \mathrm{VAl}$ alloy, ${ }^{10)}$ which has been recently reported to possess the largest Seebeck coefficient among the p-type $\mathrm{Fe}_{2} \mathrm{VAl}$ based alloys. Therefore, the

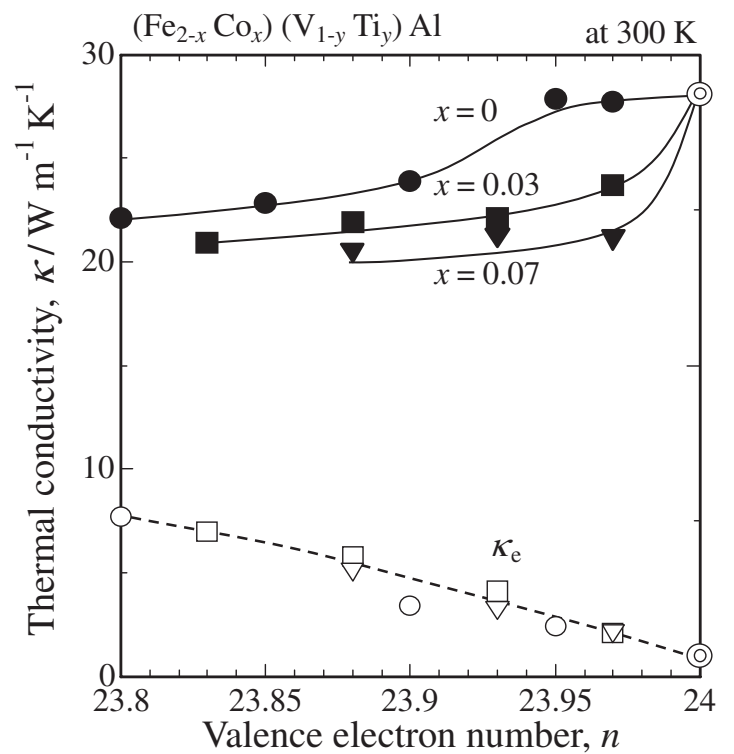

Fig. 4 Thermal conductivity of $\left(\mathrm{Fe}_{2-x} \mathrm{Co}_{x}\right)\left(\mathrm{V}_{1-y} \mathrm{Ti}_{y}\right) \mathrm{Al}$ alloys as a function of valence electron number, $n$. The contribution of electrons, $\kappa_{\mathrm{e}}$, is indicated by the open symbols. The $\kappa$ and $\kappa_{\mathrm{e}}$ values of $\mathrm{Fe}_{2} \mathrm{VAl}$ alloy are displayed by the double circles.

Seebeck coefficient of the $\left(\mathrm{Fe}_{2-x} \mathrm{Co}_{x}\right)\left(\mathrm{V}_{1-y} \mathrm{Ti}_{y}\right) \mathrm{Al}$ alloy is comparable to the Re doped alloy in spite of using the less expensive doping elements, Co and $\mathrm{Ti}$, than Re. The peak temperature at which the maximum Seebeck coefficient occurs in the Co and Ti co-doped alloys is higher than that for the stoichiometric $\mathrm{Fe}_{2} \mathrm{VAl}$ alloy. By analogy with the case of semiconductor materials, this trend suggests that the doped alloys have a pseudogap with a larger energy gap than that of the stoichiometric $\mathrm{Fe}_{2} \mathrm{VAl}{ }^{13)}$

Figure 4 shows the change in the thermal conductivity at $300 \mathrm{~K}$ in the $\left(\mathrm{Fe}_{2-x} \mathrm{Co}_{x}\right)\left(\mathrm{V}_{1-y} \mathrm{Ti}_{y}\right) \mathrm{Al}$ alloys as a function of the valence electron number. It is clearly shown that both the increasing Co composition and decreasing valence electron number, i.e. increasing Ti composition, result in a decrease in the thermal conductivity. As compared with the thermal conductivity of the $\mathrm{Fe}_{2} \mathrm{VAl}$ alloy, there is a significant decrease of about $30 \%$ for the doped alloy with $x=0.07$ and $y=0.15(n=23.88)$. The thermal conductivity, $\kappa$, is given by a simple sum of the electronic, $\kappa_{\mathrm{e}}$, and lattice contributions, $\kappa_{\mathrm{ph}}$. The electronic contribution can be estimated using the Wiedemann-Franz law; $\kappa_{\mathrm{e}}=\left(\pi^{2} / 3\right)\left(k_{\mathrm{B}}^{2} / e^{2}\right)(1 / \rho) T$, where $k_{\mathrm{B}}$ is the Boltzmann constant, and $e$ an elementary electric charge. Since this relation is valid for metals in strictly speaking, it may not be used for the pseudogap system. However, the electrical resistivity in the $\left(\mathrm{Fe}_{2-x} \mathrm{Co}_{x}\right)$ $\left(\mathrm{V}_{1-y} \mathrm{Ti}_{y}\right) \mathrm{Al}$ alloys exhibits a metallic dependence on temperature in the relatively low temperature range (see Fig. 2), as far as the valence electron number is less than $23.93(x=0.03$ and $y \geq 0.10)$. Therefore, we assume that the Wiedemann-Franz law is applicable to the alloys with $n<23.93$. The estimated $\kappa_{\mathrm{e}}$ increases with decreasing valence electron number, and they can be plotted onto a universal curve, even though the alloy compositions are different from each other. In Fig. 4, it is clear that the decrease in the total thermal conductivity arises from a reduction of $\kappa_{\mathrm{ph}}$. The reduction of $\kappa_{\mathrm{ph}}$ is mainly due to 


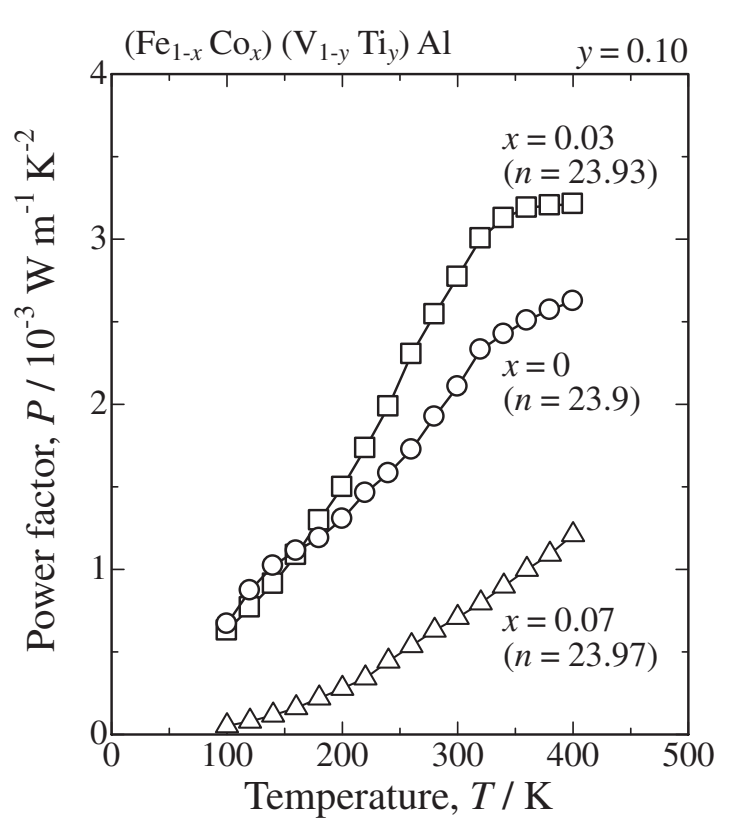

Fig. 5 Power factor of $\left(\mathrm{Fe}_{2-x} \mathrm{Co}_{x}\right)\left(\mathrm{V}_{1-y} \mathrm{Ti}_{y}\right) \mathrm{Al}$ alloys with $x=0-0.07$ and $y=0.10$ as a function of temperature. The data on the $x=0$ and $y=0.10$ alloy (open circles) are taken from the paper. ${ }^{4)}$

phonon scattering by umklapp process and geometrical effect. In general, the geometrical effect is ascribed to contributions of grain boundary, dispersion of secondary phase in a matrix and defects such as dislocations, vacancies and substitutional atoms. In the present alloys, the grain size was extremely large, i.e. several hundred $\mu \mathrm{m}$, and there was no secondary phase, so that the grain boundary and the secondary phase effects can be ignored. The effect of dislocations and vacancies may also be negligible, because the alloys were subjected to annealing at $1273 \mathrm{~K}$ for $3.6 \mathrm{ks}$ followed by furnace cooling. We believe that the effect of substitutional atoms is the most effective in reducing the thermal conductivity. Since the atomic mass of cobalt and titanium is different from iron and vanadium, respectively, the phonon could be scattered at the site of cobalt and titanium substituted. In addition, the atomic size is different between the substitutional and original atoms. The size difference generates local lattice distortion, which also scatters the phonon. However, the dominant effect of the phonon scattering caused by the substitutional atoms is not clear yet.

Figure 5 shows the temperature dependence of the power factor $\left(P=S^{2} / \rho\right)$ derived from Figs. 2 and 3 at a constant Ti composition, $y=0.10$. For comparison, the data on the $\mathrm{Fe}_{2}\left(\mathrm{~V}_{0.9} \mathrm{Ti}_{0.1}\right) \mathrm{Al}$ alloy ${ }^{4}(x=0)$ exhibiting the largest $P$ value in the Ti doped alloys are also shown in Fig. 5. The power factor of the $x=0.03$ and $y=0.10$ alloy is higher than that of the $x=0$ and $y=0.10$ alloy, and its maximum value is $3.2 \times 10^{-3} \mathrm{~W} / \mathrm{m} \mathrm{K}^{2}$, which is improved by $20 \%$ of the $x=0$ and $y=0.10$ alloy. The obtained power factor is comparable to that the p-type $\mathrm{Bi}$-Te system processed by powder metallurgy method. ${ }^{14,15)}$ However, the further Co doping $(x=0.07$ and $y=0.10)$ leads to a decrease in the power factor. The figure of merit $(Z=P / \kappa)$ at $300 \mathrm{~K}$ is also derived from Figs. 4 and 5. The largest $Z$ value obtained in this work is about $0.13 \times 10^{-3} \mathrm{~K}^{-1}$, which is larger than that for the Ti doped alloy. However, that is still smaller than that of the p-type Bi-Te system, because of the larger thermal conductivity than that of the $\mathrm{Bi}-\mathrm{Te}$ systems.

\section{Discussion}

To clarify the effect of Co and Ti co-doping, the electrical resitivity and the Seebeck coefficient at constant temperatures $(100$ and $300 \mathrm{~K})$ as a function of the valence electron number are shown in Fig. 6. In Fig. 6(a), the electrical resistivity can be plotted onto the universal curves at the respective temperatures. In contrast, the change in the Seebeck coefficient depends on the alloy compositions as shown in Fig. 6(b). The Seebeck coefficient of the $\left(\mathrm{Fe}_{2-x} \mathrm{Co}_{x}\right)\left(\mathrm{V}_{1-y} \mathrm{Ti}_{y}\right) \mathrm{Al}$ alloys with $x=0.03$ is higher than that of the $\mathrm{Fe}_{2}\left(\mathrm{~V}_{1-y} \mathrm{Ti}_{y}\right) \mathrm{Al}$ alloys in the range of $n=$ 23.9-23.97 at both the temperatures, so that the Co and $\mathrm{Ti}$ co-doped alloys exhibit the larger power factor than the Ti doped alloys. The Seebeck coefficient at a temperature $T$ is expressed by eq. (1). As in the case of the Seebeck coefficient, the electrical resistivity is proportional to the inverse of the DOS. From these relationships and Fig. 6, the change in the DOS structure by doping of cobalt and titanium is inferred. The Fermi level in the $\mathrm{Fe}_{2} \mathrm{VAl}$ alloy locates at the bottom of the pseudogap as shown by the vertical broken line in Fig. 7(a). In the previous studies, it is confirmed that the shift of the Fermi level by fourth element doping or off-stoichiometry exhibits a rigid-bandlike behavior as schematically shown in Fig. 7(b). Therefore, both the electrical resistivity and Seebeck coefficient should be plotted onto the universal curve as a function of the valence electron number. However, in this work, the Seebeck coefficient at constant temperatures cannot be plotted onto the universal curve as shown in Fig. 6(b). Rather a significant difference in the Seebeck coefficient is observed between $\left(\mathrm{Fe}_{2-x} \mathrm{Co}_{x}\right)\left(\mathrm{V}_{1-y} \mathrm{Ti}_{y}\right) \mathrm{Al}$ and $\mathrm{Fe}_{2}\left(\mathrm{~V}_{1-y} \mathrm{Ti}_{y}\right) \mathrm{Al}$ alloys. These suggest that the magnitude of the DOS is almost the same between the $\mathrm{Co}$ and $\mathrm{Ti}$ co-doped and the Ti doped alloys, but the value of $\partial N(E) / \partial E$ in the $\left(\mathrm{Fe}_{2-x} \mathrm{Co}_{x}\right)\left(\mathrm{V}_{1-y} \mathrm{Ti}_{y}\right) \mathrm{Al}$ alloys with $x=0.03$ could be larger than that in the $\mathrm{Fe}_{2}\left(\mathrm{~V}_{1-y} \mathrm{Ti}_{y}\right) \mathrm{Al}$ alloys in the range of $n=23.9-23.97$. The further doping of cobalt and titanium leads to a decrease in the Seebeck coefficient at the same valence electron number (see Fig. 3), so that the large amount of doping might cause a gentle slope in the DOS structure. The DOS structure of the $\mathrm{Fe}_{2} \mathrm{TiAl}$ Heusler alloy is similar to that of the $\mathrm{Fe}_{2} \mathrm{VAl}$ alloy in the valence band in the vicinity of the Fermi level, but the Fermi level substantially shifts to a lower energy position. ${ }^{16)}$ This may suggest that the doping of $\mathrm{Ti}$ causes a rigid-band-like shift of the Fermi level. Thus, the expected change in the DOS structure might be due to the effect of Co doping into the $\mathrm{Fe}$ site dominantly rather than that of Ti doping into the $\mathrm{V}$ site.

\section{Conclusion}

We have shown that the $\left(\mathrm{Fe}_{2-x} \mathrm{Co}_{x}\right)\left(\mathrm{V}_{1-y} \mathrm{Ti}_{y}\right) \mathrm{Al}$ alloys are attractive as a p-type thermoelectric material. The co-doping of Co and Ti effectively enhances the Seebeck coefficient up 

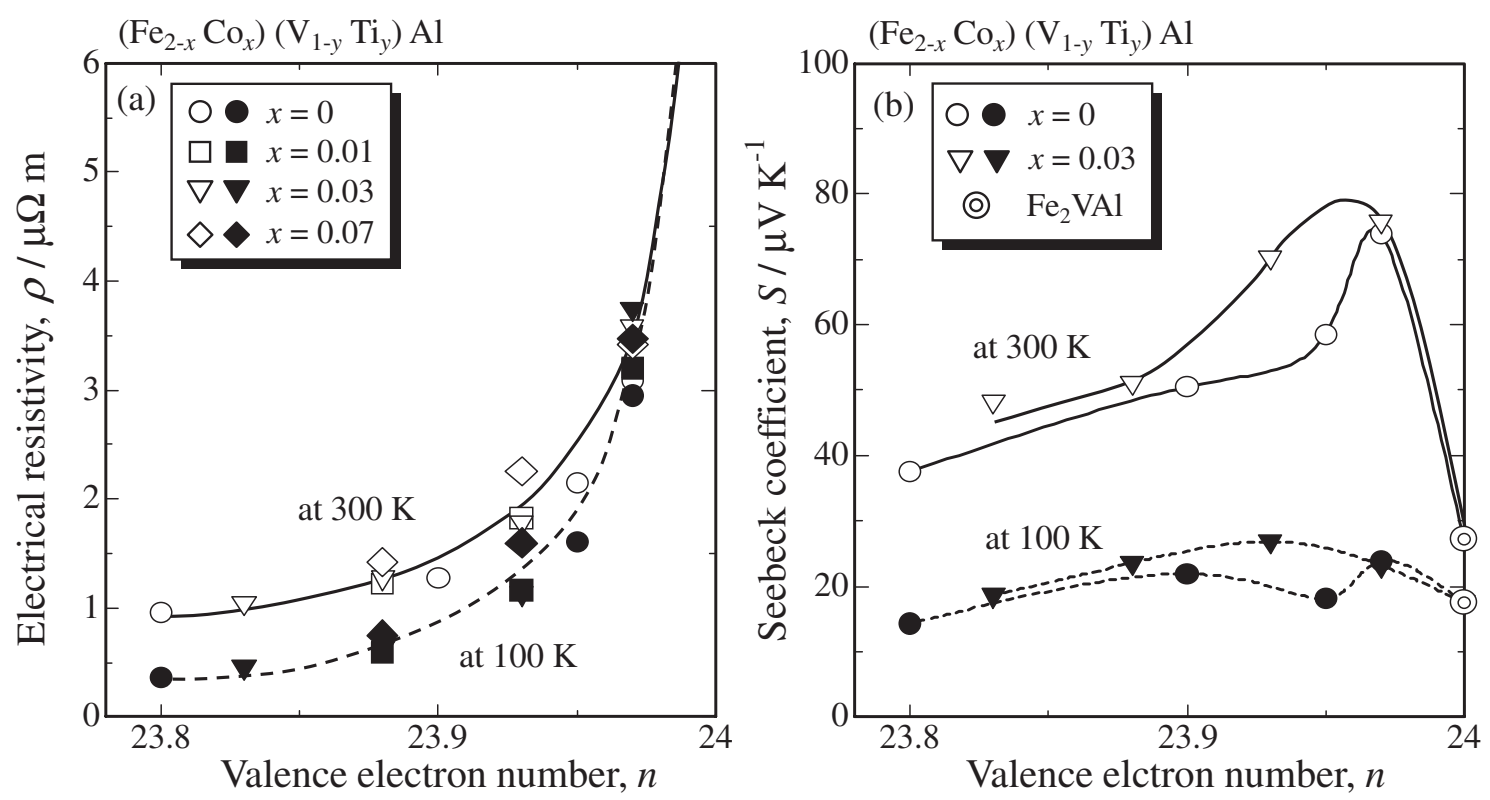

Fig. 6 Change in (a) electrical resistivity, and (b) Seebeck coefficient of $\left(\mathrm{Fe}_{2-x} \mathrm{Co}_{x}\right)\left(\mathrm{V}_{1-y} \mathrm{Ti}_{y}\right) \mathrm{Al}$ alloys at 100 and $300 \mathrm{~K}$ as a function of valence electron number.

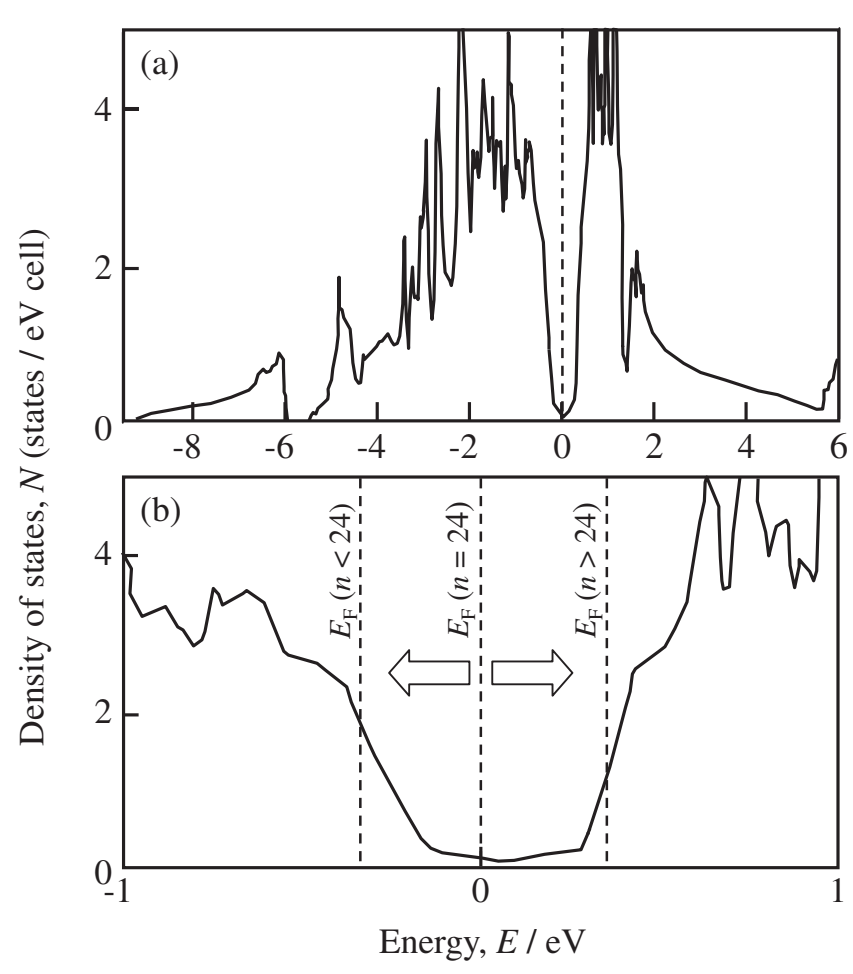

Fig. 7 (a) Total density of states in $\mathrm{Fe}_{2} \mathrm{VAl}$ alloy and (b) enlarged structure of (a) around the Fermi level, $E_{\mathrm{F}}{ }^{1)}$ The Fermi level is denoted by the broken line.

to $85 \mu \mathrm{V} / \mathrm{K}$ at $400 \mathrm{~K}$ for the alloy composition of $x=0.03$ and $y=0.10$. This value is actually larger than that for the $\mathrm{Fe}_{2}\left(\mathrm{~V}_{1-y} \mathrm{Ti}_{y}\right) \mathrm{Al}$ alloy, whose maximum value was reported to be about $80 \mu \mathrm{V} / \mathrm{K}$. The co-doping of Co and Ti effectively reduced the thermal conductivity, mainly due to phonon scattering, whose value was about $20 \mathrm{~W} / \mathrm{m} \mathrm{K}$ in the $x=0.07$ and $y=0.15$ alloy. The power factor reaches $3.2 \times 10^{-3} \mathrm{~W} /$ $\mathrm{m} \mathrm{K}^{2}$ in the alloy composition of $x=0.03$ and $y=0.10$, which is comparable to that of p-type Bi-Te thermoelectric materials processed by powder metallurgy. We expect that the gradient of the DOS structure of the co-doped alloys more sharply changes at the Fermi level than that of the stoichiometric $\mathrm{Fe}_{2} \mathrm{VAl}$ alloy in the valence band side. This change would be dominantly caused by the Co doping in the co-doped alloys.

\section{REFERENCES}

1) G. Y. Guo, G. A. Botton and Y. Nishino: J. Phys: Condens. Matter. 10 (1998) L119-L126.

2) Y. Hanada, R. O. Suzuki and K. Ono: J. Alloy Compd. 329 (2001) 63-68.

3) Y. Nishino, H. Kato, M. Kato and U. Mizutani: Phys. Rev. B 63 (2001) 233303/1-4.

4) H. Matsuura, Y. Nishino, U. Mizutani and S. Asano: J. Japan Inst. Metals 66 (2002) 767-771.

5) Y. Nishino: Recent Res. Devel: Mat. Sci. Eng 1 (2002) 557-583.

6) Y. Nishino: Mater. Sci. Forum. 449-452 (2004) 909-912.

7) K. Soda, H. Murayama, K. Shimba, S. Yagi, J. Yuhara, T. Takeuchi, U. Mizutani, H. Sumi, M. Kato, H. Kato, Y. Nishino, A. Sekiyama, S. Suga, T. Matsushita and Y. Saitoh: Phys. Rev. B 71 (2005) 245112/1-6.

8) Y. Nishino, S. Deguchi and U. Mizutani: Phys. Rev. B 74 (2006) 115115/1-6.

9) C. S. Lue, C. F. Chen, J. Y. Lin, Y. T. Yu and Y. K. Kuo: Phys. Rev. B 75 (2007) 064204/1-6.

10) F. Kobayashi, N. Ide and Y. Nishino: J. Japan Inst. Metals 71 (2006) 208-212.

11) N. F. Mott and H. Jones: The Theory of the Properties of Metals and Alloys, (Clarendon, Oxford, 1936).

12) Y. Nishino: The Science of Complex Alloy Phases, ed by T. B. Massalski and P. E. A. (Turch, TMS, 2005) 325-344.

13) H. J. Goldsmid and J. W. Sharp: J. Electronic Mater. 28 (1999) 869-872.

14) T. Sung Oh, D. B. Hyun and N. V. Kolomoets: Scripta Mater. 42 (2000) 849-854.

15) J. L. Cui, H. F. Xue and W. J. Xiu: Mater. Sci. Eng. B 135 (2006) 44-49.

16) G. A. Botton, Y. Nishino and C. J. Humphreys: Intermetallics 8 (2000) 1209-1214. 